\title{
The Weyl Transformation and Quantisation for Locally Compact Abelian Groups
}

By

\author{
Mark A. HENNINGS*
}

\begin{abstract}
Lunn's method of constructing a Weyl transformation $W$ to act on all the tempered distributions $\mathscr{S}^{\prime}\left(\boldsymbol{R}^{2 n}\right)$ over $\boldsymbol{R}^{2 n}$ is considered. Ideas of Hudson, Peck and Wilkinson are used to extend this construction to provide a Weyl transformation acting on the whole of $\mathscr{S}^{\prime}(G)$ for any phase space $G$, where $G$ is a locally compact separable abelian group such that $x \mapsto \rightarrow 2 x$ is an automorphism of $G$. Lunn's construction is also generalised directly to provide a Weyl transformation for the whole of $\mathscr{S}^{\prime}(G)$ where $G=C \times \hat{C}$ is the phase space associated with the configuration space $C$ (a locally compact separable abelian group for which the map $x \mapsto \rightarrow 2 x$ is an automorphism).

Finally, these two different formulations of the Weyl transformation are compared, and algebras of physical observables $\mathcal{O}_{m}(G)$ and $\mathcal{O}(G)$ are found. It is shown that $\mathcal{O}(G)=$ $\mathcal{O}_{m}(G) \cap \mathcal{O}_{m}(G)^{*}$, where* is the natural involution on $S^{\prime}(G)$, and that the algebra structures of $\mathcal{O}(G)$ and $\mathcal{O}_{m}(G)$ coincide on $\mathcal{O}(G)$. Hence both formulations of the Weyl transformation provide the same algebra of physical observables.
\end{abstract}

\section{§1. Introductory}

Lunn [10] has developed an extension of the Weyl transformation for the group $\boldsymbol{R}^{2 n}$. Using the antisymmetric bicharacter

$$
\sigma(x, y)=\exp \left(\frac{i}{2 \hbar}\left[x_{1} \circ y_{2}-x_{2} \cdot y_{1}\right]\right) \quad x=\left(x_{1}, x_{2}\right), \quad y=\left(y_{1}, y_{2}\right)
$$

and the unitary $\sigma$-representation $U$ of $\boldsymbol{R}^{2 n}$ on $L^{2}\left(\mathbb{R}^{n}\right)$ given by

$$
[U(x) f](q)=\exp \left(\frac{i}{\hbar} x_{2}\left(q-\frac{1}{2} x_{1}\right)\right) f\left(q-x_{1}\right)
$$

she obtains a bijection $W: \mathscr{S}^{\prime}\left(\boldsymbol{R}^{2 n}\right) \rightarrow \mathscr{L}\left(\mathscr{S}\left(\boldsymbol{R}^{n}\right), \mathscr{S}^{\prime}\left(\boldsymbol{R}^{n}\right)\right)$, the space of weakly continuous linear maps from $\mathscr{S}\left(\boldsymbol{R}^{n}\right)$ to $\mathscr{S}^{\prime}\left(\boldsymbol{R}^{n}\right)$, whose restriction to $\mathscr{S}\left(\boldsymbol{R}^{2 n}\right)$ is the traditional Weyl transformation for Schwartz functions. In [5] the author has identified a ${ }^{*}$-algebra $\mathcal{O}\left(\boldsymbol{R}^{2 n}\right)$ contained in

Communicated by H. Araki, May 22, 1985.

* St. John's College, Oxford, OX1 3JP, England. 
$\mathscr{S}^{\prime}\left(\boldsymbol{R}^{2 n}\right)$, and has shown that there is essentially only one strictly irreducible representation of this algebra, and that this is given by the Weyl transformation $W$, since for every $u \in \mathcal{O}\left(\mathbb{R}^{2 n}\right)$ the map $W(u)$ may be regarded as a continuous linear map from $\mathscr{S}\left(\mathbb{R}^{n}\right)$ to itself which possesses an adjoint with respect to the natural preinner product structure of $\mathscr{S}\left(\mathbb{R}^{n}\right)$.

If we consider the twisted Fourier transformation $\mathscr{F}_{\sigma}$ from $\mathscr{S}\left(\mathbb{R}^{2 n}\right)$ to itself given by

$$
\left(\mathscr{F}_{\sigma} f\right)(x)=h^{-n} \iint_{R^{2 n}} f(y) \sigma(x, y)^{2} d y
$$

and lift $\mathscr{F}_{\sigma}$ to a topological isomorphism from $\mathscr{S}^{\prime}\left(\mathbb{R}^{2 n}\right)$ to itself, then the Weyl transformation $W$ can be decomposed into a composition of two operators $W=U \cdot \mathscr{F}_{0}$, where $U: \mathscr{S}^{\prime}\left(\mathbb{R}^{2 n}\right) \rightarrow \mathscr{L}\left(\mathscr{S}\left(\mathbb{R}^{n}\right), \mathscr{S}^{\prime}\left(\mathbb{R}^{n}\right)\right)$ is the bijection which has the weakly convergent formulation

$$
U(v)=h^{-n} \iint_{R^{2 n}} \mathrm{v}(x) U(x) d x \quad v \in \mathscr{S}^{\prime}\left(\boldsymbol{R}^{2 n}\right) .
$$

In this work we shall be interested in studying ways of extending this construction to provide a Weyl transformation for more general phase spaces than $\mathbb{R}^{2 n}$.

\section{§2. A Generalised Fourier-Plancherel Theorem}

Hudson et al. [6] have considered a locally compact abelian group $G$ equipped with a multiplier $\sigma$, and have found a generalised form of the Fourier-Plancherel theorem which is compatible with the multiplier $\sigma$. This has been done by considering the associated multiplier $\omega(x, y)=\sigma(y, x)^{-1}$, and the regular $\omega$-representation $R_{\omega}$ of $G$ on $L^{2}(G)$

$$
\left[R_{\omega}(x) f\right](y)=\omega(y, x)^{-1} f(y+x) .
$$

Then $L^{1}(G) \cap L^{2}(G)$ becomes a Hilbert algebra when equipped with its natural preinner product structure, multiplication and involution being given by

$$
\begin{aligned}
& (f \sigma g)(x)=\int_{G} f(y) \sigma(y, x-y)^{-1} g(x-y) d y \\
& f^{\sigma}(x)=\sigma(x,-x) \overline{f(-x)} .
\end{aligned}
$$


For any $f \in L^{1}(G) \cap L^{2}(G)$, we shall denote by $\lambda_{\sigma}(f)$ the bounded linear operator on $L^{2}(G)$ which is the closure of the map $g \hookrightarrow f \sigma g$. If $N_{\omega}$ is the von Neumann algebra generated by the operators $R_{\omega}(x)$, then there exists a translation-invariant faithful normal semi-finite trace $\tau$ on $N_{\omega}$ which is unique to within normalisation. If we let $L^{2}\left(N_{\omega}, \tau\right)$ be the Hilbert space generated by $N_{\omega}$ and $\tau$, then for any $f \in L^{1}(G)$ $\cap L^{2}(G)$ the map $\lambda_{\sigma}(f) \in L^{2}\left(N_{\omega}, \tau\right)$ has the weakly convergent formulation

$$
\lambda_{\sigma}(f)=\int_{G} f(x) R_{\omega}(x) * d x
$$

and, for a suitable normalisation of $\tau$, the map $f \mapsto \lambda_{\sigma}(f)$ may be extended to a unitary map $U: L^{2}(G) \rightarrow L^{2}\left(N_{\omega}, \tau\right)$.

The close similarity between equations (4) and (8) leads us to look for ways of relating these two approaches, and using and extending the formalisms of Hudson et al. to provide a generalised Weyl transformation for a physical system with phase space given by the group $G$. This is the aim of the work in the next two sections of this paper. We shall simplify our notation, eliminating the need for the multiplier $\omega$, by observing that

$$
\left\langle g, R_{\omega}(x) f\right\rangle=\left(f \sigma g^{\sigma}\right)(x)
$$

for $f, g \in L^{2}(G)$. Thus if we define the function $R_{f g} \in L^{2}(G)$ for any $f, g \in L^{2}(G)$ by

$$
R_{f g}(x)=\left\langle f, R_{\omega}(x) *_{g}\right\rangle
$$

then the map $U(h)$ is defined for $h \in L^{1}(G) \cap L^{2}(G)$ by

$$
\langle f, U(h) g\rangle=\int_{G} h(x) R_{f g}(x) d x=\left\langle f \sigma g^{\sigma}, h\right\rangle \quad f, g \in L^{2}(G) .
$$

Equation (11) is what we shall use to extend our notion of the Weyl transformation.

\section{§3. The Schwartz-Bruhat Functions}

Unfortunately, the results of $\S 2$ cannot be used immediately in the required manner, because the twisted Fourier transformation $\mathscr{F}_{\sigma}$ cannot be applied to the space $L^{2}(G)$ very conveniently.

The normal Fourier transformation of a function $f \in L^{1}(G)$ is 


$$
\hat{f}(\xi)=\int_{G} f(x)\langle x, \xi\rangle d x \quad \xi \in \hat{G}
$$

where $\hat{G}$ is the topological dual of $G$. If we assume that $G$ is separable and that the multiplier is strongly nondegenerate as in [6], so that the $\operatorname{map} \xi: G \rightarrow \hat{G}$ defined by

$$
\langle y, \xi x\rangle=\tilde{\sigma}(x, y)=\sigma(x, y) \sigma(y, x)^{-1} \quad x, y \in G
$$

is bijective, then $\xi$ becomes a topological isomorphism [3], and we can define a twisted Fourier transformation $\mathscr{F}_{\sigma}$ on $L^{1}(G)$ by setting

$$
\left(\mathscr{F}_{\sigma} f\right)(x)=\hat{f}(\xi x)=\int_{G} f(y)\langle y, \xi x\rangle d x=\int_{G} f(y) \tilde{\boldsymbol{\sigma}}(x, y) d y
$$

which agrees with the definition used in $\S 1$. However, it is difficult to describe precisely for what functions $f \in L^{1}(G)$ it is true that $\mathscr{F}_{\sigma} f \in L^{2}(G)$, and so it is more natural to restrict our attention to the Schwartz-Bruhat functions $\mathscr{S}(G)$ as defined in [1], [12], in which case $\mathscr{F}_{\sigma}$ becomes a topological isomorphism from $\mathscr{S}(G)$ to itself, and since $\tilde{\sigma}$ is an antisymmetric bicharacter we have that $\mathscr{F}_{\sigma}^{2}=I$ for a suitable normalisation of the Haar measure on $G$. If we consider the topological isomorphism $S: \mathscr{S}(G) \rightarrow \mathscr{S}(G)$ defined by $(S f)(x)=f(-x)$ for $f \in \mathscr{S}(G)$, then $\mathscr{F}_{\sigma}$ may be extended to a topological isomorphism from $\mathscr{S}^{\prime}(G)$ to itself given by

$$
\left(\mathscr{F}_{\sigma} u, f\right)=\left(u, \mathscr{F}_{\sigma} S f\right)=\left(u, S \mathscr{F}_{0} f\right) \quad u \in \mathscr{S}^{\prime}(G), f \in \mathscr{S}(G)
$$

such that $\mathscr{F}_{\sigma}^{2}=I$. Thus if we can find a suitable map $U$ defined on the whole of $\mathscr{S}^{\prime}(G)$ we can compose these two maps to obtain a Weyl transformation $W$.

\section{§4. Construction of the Map $U$}

Let us now suppose that $\sigma$ is a strongly nondegenerate multiplier on the locally compact separable abelian group $G$, and let $\xi: G \rightarrow \hat{G}$ be the associated isomorphism. As in [3], [4], [12], for any subset $E$ of $G$ we define

$$
(E \sigma)=E^{\xi}=\{g \in G \mid \tilde{\sigma}(s, g)=1 \quad \forall s \in E\}
$$

whence $(E \sigma)$ is a closed subgroup of $G$. If $H$ is a closed subgroup of $G$ then

$$
\xi(H \sigma)=H^{\perp} \quad \xi H=(H \sigma)^{\perp} \quad((H \sigma) \sigma)=H .
$$


A subgroup $H$ of $G$ is called isotropic if $H \subseteq(H \sigma)$. Hannabuss [4] has shown that $\mathscr{S}(G)$ is the inductive limit of the spaces $\mathscr{S}((L \sigma), L)$, where $L$ runs over all compact isotropic subgroups of $G$ such that $(L \sigma) / L$ is elementary.

Let us now assume that $\sigma$ is itself an antisymmetric bicharacter, so that $\tilde{\sigma}=\sigma^{2}$. This is not an unreasonable assumption, because it is shown in [9] that for a large class of groups $G$ every multiplier is similar to an antisymmetric bicharacter. In particular this is true if the map $x \mapsto 2 x$ is an automorphism of $G$. If we now define $\zeta: G \rightarrow \hat{G}$ by

$$
\langle y, \zeta x\rangle=\sigma(x, y) \quad x, y \in G
$$

then it is easy to prove that $\zeta$ is also a group isomorphism. For any subset $E$ of $G$ we can define the closed subgroup $E^{\zeta}$ by the analogous formula to (16), and results analogous to those in (17) hold here. Also $E^{\zeta} \subseteq(E \sigma)$ for any set $E$.

Proposition 1. If $(H, K)$ is an admissible pair for $G$, then $K / K \cap H^{\zeta}$ is an elementary group.

Proof. [12].

Proposition 2. If $((L \sigma), L)$ is an admissible pair, then $\left((L \sigma),(L \sigma)^{\zeta}\right)$ is also admissible, and $(L \sigma)^{\zeta}$ is a compact subgroup of finite index in $L$.

Proof. ( $L \sigma)$ is open, so that $(L \sigma)^{\zeta}$ is compact. $(L \sigma)^{\zeta} \subseteq((L \sigma) \sigma)=L$, so that $(L \sigma)^{\zeta}$ is a compact subgroup of $L$. Thus $(L \sigma)^{\zeta}=L \cap(L \sigma)^{\zeta}$, so that $L /(L \sigma)^{\zeta}$ is elementary. Hence $\left((L \sigma),(L \sigma)^{\zeta}\right)$ is admissible.

If $x \in L$, then $\sigma(2 x, y)=\sigma(x, y)^{2}=\tilde{\sigma}(x, y)=1$ for all $y \in(L \sigma)$, so that $2 x \in(L \sigma)^{\zeta}$. Thus every element of $L /(L \sigma)^{\zeta}$ has order 1 or 2 . Since $L /(L \sigma)^{b}$ is compact and elementary, it is isomorphic to $T^{q} \times \Gamma$ for some $q \geqq 0$ and some finite group $\Gamma$. But every element of $L /(L \sigma)^{\zeta}$ has order 1 or 2, so that $q=0$ and hence $L /(L \sigma)^{\zeta} \equiv \Gamma$ is finite.

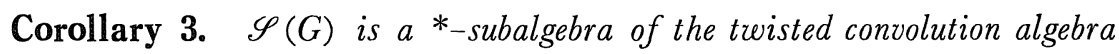
$L^{1}(G)$.

Proof. [4]. Proposition 2 has been proved in detail in order to 
clarify some points of obscurity in the proof of [4].

Theorem 4. The map $\sigma: \mathscr{S}(G) \otimes \mathscr{S}(G) \rightarrow \mathscr{S}(G)$ is separately continuous.

Proof. For any $f \in \mathscr{S}(G)$ and any admissible pair $(H, K)$, we can find a compact isotropic subgroup $L$ of $G$ such that $(L \sigma) / L$ is elementary and with $f \in \mathscr{S}\left((L \sigma),(L \sigma)^{\zeta}\right)$ and $\mathscr{S}(H, K) \subseteq \mathscr{S}\left((L \sigma),(L \sigma)^{\zeta}\right)$. The known properties of Schwartz functions and bicharacters of elementary groups tell us that the two linear maps from $\mathscr{S}\left((L \sigma),(L \sigma)^{\zeta}\right)$ to itself given by $g \mapsto f \sigma g$ and $g \mapsto g \sigma f$ restrict to continuous linear maps $\tilde{g} \mapsto(f \sigma g) \sim$ and $\tilde{g} \mapsto(g \sigma f) \sim$ from $\mathscr{S}\left((L \sigma) /(L \sigma)^{\xi}\right)$ to itself, and hence they are themselves continuous. Thus the two linear maps $g \mapsto f \sigma g$ and $g \mapsto g \sigma f$ from $\mathscr{S}(G)$ to itself are both continuous when restricted to $\mathscr{S}((L \sigma)$, $\left.(L \sigma)^{\zeta}\right)$, and so are also continuous when restricted to $\mathscr{S}(H, K)$. Since $\mathscr{S}(G)$ is the inductive limit of the spaces $\mathscr{S}(H, K)$ we deduce that the maps $g \mapsto f \sigma g$ and $g \mapsto g \sigma f$ are continuous on the whole of $\mathscr{S}(G)$, as required.

Now consider the space $\mathscr{G}(G)$ of Gaussian functions as introduced in [7]. Elementary calculations enable us to prove that

Proposition 5. $\sigma(\mathscr{G}(G) \otimes \mathscr{G}(G))=\mathscr{G}(G)$.

Thus $\mathscr{G}(G) \subseteq \sigma(\mathscr{S}(G) \otimes \mathscr{S}(G)) \subseteq \mathscr{S}(G)$, and so the fact that $\mathscr{G}(G)$ is dense in $\mathscr{S}(G)$ tells us that $\sigma(\mathscr{S}(G) \otimes \mathscr{S}(G))$ is dense in $\mathscr{S}(G)$.

We are at last in a position to calculate the required map $U$. For any $g \in \mathscr{S}(G)$ and $v \in \mathscr{S}^{\prime}(G)$, consider the map $U(v) g: \mathscr{S}(G) \rightarrow \boldsymbol{C}$ given by

$$
[U(v) g](f)=\left(v, S\left(g \sigma f^{\sigma}\right)\right) \quad f \in \mathscr{S}(G) .
$$

We now see that $U(v) g$ is a continuous antilinear functional on $\mathscr{S}(G)$; in other words $U(v) g \in \mathscr{S}^{*}(G)$, the conjugate dual of $\mathscr{S}(G)$. It is also clear that we have defined a weakly continuous linear map $U(v)$ from $\mathscr{S}(G)$ to $\mathscr{S}^{*}(G)$ such that

$$
(U(v) g, f \sigma h)^{*}=\left(v, S\left(g \sigma(f \sigma h)^{\sigma}\right)\right)=\left(v, S\left(\left(g \sigma h^{\sigma}\right) \sigma f^{\sigma}\right)\right)=\left(U(v)\left(g \sigma h^{\sigma}\right), f\right)^{*}
$$


for any $f, g, h \in \mathscr{S}(G)$. If we define $\mathscr{L}_{\sigma}(G)$ to be the space of all maps $A$ in $\mathscr{L}\left(\mathscr{S}(G), \mathscr{S}^{*}(G)\right)$ such that

$$
(A g, f \sigma h)^{*}=\left(A\left(g \sigma h^{\sigma}\right), f\right)^{*} \quad f, g, h \in \mathscr{S}(G)
$$

then we have defined a linear map $U: \mathscr{S}^{\prime}(G) \rightarrow \mathscr{L}_{\sigma}(G)$.

\section{Proposition 6. The map $U$ is injective.}

Proof. If $v \in \mathscr{S}^{\prime}(G)$ and $U(v)=0$, then $\left(v, S\left(g \sigma f^{\circ}\right)\right)=0$ for all $f, g$ $\in \mathscr{S}(G)$. Since $\sigma(\mathscr{S}(G) \otimes \mathscr{S}(G))$ is dence in $\mathscr{S}(G)$, we deduce that $S \sigma(\mathscr{S}(G) \otimes \mathscr{S}(G))$ is also dense in $\mathscr{S}(G)$, and so $v=0$ and hence $U$ is injective.

Thus we can combine $U$ with $\mathscr{F}_{\sigma}$ to obtain an injective Weyl transformation $W: \mathscr{S}^{\prime}(G) \rightarrow \mathscr{L}_{\sigma}(G)$. It would be desirable to show that the map $U$ is also bijective, but there are many technical difficulties inherent in attempting to establish this. For example, if $G$ were such that for every compact isotropic subgroup $L$ for which $(L \sigma) / L$ is elementary the space $\mathscr{S}\left((L \sigma),(L \sigma)^{\zeta}\right)$ possessed a bounded approximate identity, then $U$ and $W$ would be bijective. However, the fact that $\mathscr{S}(G)$ is not metrisable in general, and the uncertainty as to whether the multiplication $\sigma: \mathscr{S}(G) \otimes \mathscr{S}(G) \rightarrow \mathscr{S}(G)$ is jointly continuous or not, makes it highly unlikely that the above condition is true, and greatly hinders any other approach to answering the question.

We can, however, make some progress. Equation (19) not only defines the map $U$, but also defines the inverse map $U(v) \mapsto v$ by the density of $\sigma(\mathscr{S}(G) \otimes \mathscr{S}(G))$ in $\mathscr{S}(G)$. Since $\sigma$ is an antisymmetric bicharacter, $\omega=\sigma$, and so if we consider the linear map $\rho_{x}: \mathscr{S}(G) \rightarrow$ $\mathscr{S}(G)$ given by

$$
\left(\rho_{x} f\right)(y)=\sigma(x, y) f(x-y)=\left[R_{\omega}(-y) f\right](x)=\left[R_{\omega}(y)^{*} f\right](x)
$$

for $x \in G$ and $f \in \mathscr{S}(G)$, and if we define $\mathcal{O}_{c}(G)$ to be the space of all $v \in \mathscr{S}^{\prime}(G)$ such that the map $f \mapsto v \sigma_{1} f$ is a continuous linear map from $\mathscr{S}(G)$ to itself, where

$$
\left(v \sigma_{1} f\right)(x)=\left(v, \rho_{x} f\right)
$$

then, for $v \in \mathcal{O}_{c}(G), U(v)$ may be regarded as the continuous linear map from $\mathscr{S}(G)$ to itself given by $U(v) g=v \sigma_{1} g$. It is also clear that $U(v)=\lambda_{\sigma}(v)$ for any $v \in \mathscr{S}(G)$, so that $\mathscr{S}(G) \subseteq \mathcal{O}_{c}(G)$ and the various 
convolution operators $\sigma$ are the same. It may be shown that $\mathcal{O}_{c}(G)$ has a multiplicative structure given by $(u, v) \mapsto u \sigma_{2} v$ where

$$
\left(u \sigma_{2} v, f\right)=\left(u, S\left(v \sigma_{1} S f\right)\right) \quad u, v \in \mathcal{O}_{c}(G), f \in \mathscr{S}(G)
$$

and we have that $U\left(u \sigma_{2} v\right)=U(u) U(v)$ for all $u, v \in \mathcal{O}_{c}(G)$. Hence $\mathcal{O}_{m}(G)=\mathscr{F}_{\sigma} \mathcal{O}_{c}(G)$ becomes an algebra with respect to the induced multiplicative structure

$$
u^{*}{ }_{2} v=\mathscr{F}_{\sigma}\left[\left(\mathscr{F}_{\sigma} u\right) \sigma_{2}\left(\mathscr{F}_{\sigma} v\right)\right] \quad u, v \in \mathcal{O}_{m}(G)
$$

and the Weyl transformation $W$ provides a representation of the algebra $\mathcal{O}_{m}(G)$. In the case $G=\boldsymbol{R}^{2 n}$, the algebras $\mathcal{O}_{c}\left(\boldsymbol{R}^{2 n}\right)$ and $\mathcal{O}_{m}\left(\boldsymbol{R}^{2 n}\right)$ are precisely the usual algebras of distributions of weak decrease discussed in [8] and [14].

Finally, we notice that if we define the $\operatorname{map} *_{1}: \mathcal{O}_{m}(G) \times \mathscr{S}(G) \rightarrow$ $\mathscr{S}(G)$ by the formula $v^{*} f=\mathscr{F}_{\sigma}\left[\left(\mathscr{F}_{\sigma} v\right) \sigma_{1}\left(\mathscr{F}_{\sigma} f\right)\right]$ for $v \in \mathcal{O}_{m}(G)$ and $f \in$ $\mathscr{S}(G)$, then the convolutions $*_{1}$ and $*_{2}$ on $\mathcal{O}_{m}(G)$ are more simply related than are $\sigma_{1}$ and $\sigma_{2}$ on $\mathcal{O}_{c}(G)$, since elementary calculations show that

$$
\left(u^{*}{ }_{2} v, f\right)=\left(u, v^{*}{ }_{1} f\right) \quad u, v \in \mathcal{O}_{m}(G), f \in \mathscr{S}(G) .
$$

\section{§5. The Weyl Transformation for a Configuration Space $C$}

We shall now attempt to generalise directly the constructions of Lunn [10] outlined in $\S 1$, and then compare our results with the general ones of $\S 4$.

In cases of physical interest, we naturally want our observables to be functions on some phase space $G$. Let us now assume in particular that our physical system has phase space $G$ generated by a configuration space $C$, where $C$ is a locally compact separable abelian group. The phase space $G$ will then be given by $C \times C$. It is well known that there is essentially only one antisymmetric bicharacter $\sigma$ on $G$ for which both $C$ and $\hat{C}$ are isotropic, and that is given by

$$
\sigma((x, \xi),(y, \eta))=\langle x, \eta\rangle\langle y, \xi\rangle^{-1} \quad(x, \xi),(y, \eta) \in G .
$$

Now $\sigma$ is an antisymmetric bicharacter, and will be strongly nondegenerate if and only if $x \mapsto 2 x$ is an automorphism of $C$.

For any locally compact separable abelian group $C$, let $\mathscr{A}$ denote the family of all admissible pairs of subgroups of $C$, and let $\hat{\mathscr{A}}$ denote 
the family of all admissible pairs of subgroups of $\hat{C}$. There is of course a one-to-one correspondence between $\mathscr{A}$ and $\hat{\mathscr{A}}$, since $(H, K)$ $\in \mathscr{A}$ if and only if $\left(K^{\perp}, H^{\perp}\right) \in \hat{\mathscr{A}}$. If $H$ is a compactly generated open subgroup of $C$, then we can define the nonempty sets ([12]):

$$
\mathscr{A}_{H}=\{K \subseteq C \mid(H, K) \in \mathscr{A}\} \quad \hat{\mathscr{A}}_{H}=\left\{L \subseteq \hat{C} \mid\left(L, H^{\perp}\right) \in \hat{\mathscr{A}}\right\}
$$

and observe that $K \in \mathscr{A}_{H}$ if and only if $K^{\perp} \in \hat{\mathscr{A}}_{H}$.

Proposition 7. $\mathscr{A}_{H}$ has trivial intersection for any compactly generated open subgroup $H$ of $C$.

Proof. For any $L \in \hat{\mathscr{A}}_{H}$ and any $\xi \in \hat{C}$, we know that $\left(L+\langle\xi\rangle, H^{\perp}\right)$ $\in \hat{\mathscr{A}}([12])$, so that $L+\langle\xi\rangle \in \hat{\mathscr{A}}_{H}$. Thus $\hat{C}=\cup_{L \in \hat{A}_{H}} L=\cup_{K \in \mathscr{A}_{H}} K^{\perp}$. Hence, if $x \in \cap_{K \in \mathscr{A}_{H}} K$, then $\langle x, \xi\rangle=1$ for all $\xi \in \hat{C}$ so that $x=0$.

Theorem 8. If $C_{1}$ and $C_{2}$ are two locally compact separable abelian groups with associated families of admissible pairs $\mathscr{A}_{1}$ and $\mathscr{A}_{2}$, then $\mathscr{S}\left(C_{1}\right.$ $\left.\times C_{2}\right)$ is the inductive limit of the spaces $\mathscr{S}\left(H_{1} \times H_{2}, K_{1} \times K_{2}\right)$ where $\left(H_{1}\right.$, $\left.K_{1}\right) \in \mathscr{A}_{1}$ and $\left(H_{2}, K_{2}\right) \in \mathscr{A}_{2}$.

Proof. Consider the continuous open canonical group homomorphisms $\pi_{i}: C_{1} \times C_{2} \rightarrow C_{i}$. If $f \in \mathscr{S}\left(C_{1} \times C_{2}\right)$, then $f \in \mathscr{S}(H, K)$ where $(H$, $K)$ is an admissible pair for $C_{1} \times C_{2}$. Define $H_{i}=\pi_{i}(H)$. Each $H_{i}$ is an open compactly generated subgroup of $C_{i}$, and $H \subseteq H_{1} \times H_{2}$. Thus $\left(H_{1} \times H_{2}, K\right)$ is admissible and $f \in \mathscr{S}\left(H_{1} \times H_{2}, K\right)$.

$\mathscr{Z}=\left\{L_{1} \times L_{2} \mid L_{i} \in \mathscr{A}_{H_{i}}\right\}$ is a collection of compact subgroups of $H_{1}$ $\times H_{2}$ whose total intersection is $\{0\}$. Hence $\{(K+L) / K \mid L \in \mathscr{Z}\}$ is a collection of compact subgroups of $H_{1} \times H_{2} / K$ whose intersection is $\{K\}$. Since $H_{1} \times H_{2} / K$ is elementary, it possesses no small subgroups, so there exists $L \in \mathscr{Z}$ such that $(K+L) / K=\{K\}$; in other words such that $L \subseteq K$. Let $L=K_{1} \times K_{2}$. Then $H_{i} / K_{i}$ are both elementary, so that $H_{1} \times H_{2} / K_{1} \times K_{2}$ is also elementary. Thus $\left(H_{1} \times H_{2}, K_{1} \times K_{2}\right)$ is admissible, with $\left(H_{i}, K_{i}\right) \in \mathscr{A}_{i}$, and since we see that $K_{1} \times K_{2} \subseteq K \subseteq H \subseteq$ $H_{1} \times H_{2}$, we deduce that $f \in \mathscr{S}\left(H_{1} \times H_{2}, K_{1} \times K_{2}\right)$.

Theorem 9. If $C_{1}$ and $C_{2}$ are two locally compact separable abelian groups, then $\mathscr{S}\left(C_{1} \times C_{2}\right) \equiv \mathscr{S}\left(C_{1}\right) \otimes \mathscr{S}\left(C_{2}\right)$. 
Proof. For $\left(H_{i}, K_{i}\right) \in \mathscr{A}_{i}$, the kernel theorem of [14] tells us that $\mathscr{D}\left(H_{1} \times H_{2} / K_{1} \times K_{2}\right) \equiv \mathscr{D}\left(H_{1} / K_{1}\right) \bar{\otimes} \mathscr{D}\left(H_{2} / K_{2}\right)$. Since the injection from $\mathscr{D}\left(H_{1} \times H_{2} / K_{1} \times K_{2}\right)$ to $\mathscr{S}\left(H_{1} \times H_{2} / K_{1} \times K_{2}\right)$ is continuous [1], it follows that $\mathscr{S}\left(H_{1} / K_{1}\right) \otimes \mathscr{S}\left(H_{2} / K_{2}\right)$ is dense in $\mathscr{S}\left(H_{1} \times H_{2} / K_{1} \times K_{2}\right)$. Since $\mathscr{S}\left(H_{i} / K_{i}\right)$ are both nuclear, we deduce that $\mathscr{S}\left(H_{1} \times H_{2} / K_{1} \times K_{2}\right) \equiv$ $\mathscr{S}\left(H_{1} / K_{1}\right) \otimes \mathscr{S}\left(H_{2} / K_{2}\right)$, and so $\mathscr{S}\left(H_{1} \times H_{2}, K_{1} \times K_{2}\right)$ is isomorphic to $\mathscr{S}\left(H_{1}, K_{1}\right) \otimes \mathscr{S}\left(H_{2}, K_{2}\right)$. We now use Proposition 14 of [2] to take inductive limits and deduce that $\mathscr{S}\left(G_{1} \times G_{2}\right)$ is indeed isomorphic to $\mathscr{S}\left(C_{1}\right) \bar{\otimes} \mathscr{S}\left(C_{2}\right)$.

We now have enough technical detail to generalise the results of $\S 1$. Consider the $\sigma$-representation $U$ of $C \times \hat{C}$ defined on $L^{2}(G)$ by the formula

$$
[U(x, \xi) f](y)=\langle 2 y-x, \xi\rangle f(y-x) \quad f \in L^{2}(C)
$$

and, for any $f, g \in L^{2}(C)$, consider the function $F_{f g}$ on $C \times \hat{C}$ given by

$$
F_{f g}(x, \xi)=\int_{C} f(y)[U(x, \xi) g](y) d y .
$$

Consider the partial Fourier transform $\mathscr{F}_{2}: \mathscr{S}(C \times G) \rightarrow \mathscr{S}(C \times \mathcal{C})$ given by

$$
\left(\mathscr{F}_{2} f\right)(x, \xi)=\int_{C} f(x, y)\langle y, \xi\rangle \mathrm{dy} .
$$

Since we are assuming that the map $x \mapsto 2 x$ is an automorphism of $C$, let us also consider the map $R: \mathscr{S}(C \times C) \rightarrow \mathscr{S}(C \times C)$ given by

$$
(R f)(x, y)=\alpha f\left(\frac{1}{2}(x+y), \frac{1}{2}(x-y)\right)
$$

where $\alpha$ is the positive constant defined by the relationship

$$
\int_{C} f(y) d y=\alpha \int_{c} f\left(\frac{1}{2} y\right) d y \quad f \in \mathscr{S}(C) .
$$

Elementary calculations show that $\mathscr{F}_{2}$ and $R$ are both topological isomorphisms, and that $F_{f g}=\mathscr{F}_{2} R(f \otimes g)$ for any $f, g \in \mathscr{S}(G)$, so that $\left\{F_{f g} \mid f, g \in \mathscr{S}(C)\right\}$ spans a dense linear subspace of $\mathscr{S}(C \times \hat{C})$.

For any $v \in \mathscr{S}^{\prime}(C \times \hat{C})$ and any $g \in \mathscr{S}(G)$, consider the map $U(v) g$ : $\mathscr{S}(C) \rightarrow \mathbb{C}$ given by

$$
[U(v) g](f)=\left(v, F_{f \mathrm{~g}}\right) \quad f \in \mathscr{S}(G) .
$$

For any $\in \mathscr{S}(G)$, the maps from $\mathscr{S}(G)$ to $\mathscr{S}(C \times G)$ given by 
$g \mapsto f \otimes g$ and $g \mapsto \mathrm{g} \otimes f$ are continuous, and so the maps from $\mathscr{S}(C)$ to $\mathscr{S}(C \times \hat{C})$ given by $\mapsto F_{f g}$ and $g \mapsto F_{g f}$ are continuous. Hence $U(v) g \in$ $\mathscr{S}^{\prime}(C)$, and we have defined a weakly continuous linear map $U(v)$ : $\mathscr{S}(G) \rightarrow \mathscr{S}^{\prime}(G)$. Thus we have defined a linear map $U: \mathscr{S}^{\prime}(G \times \hat{C})$ $\rightarrow \mathscr{L}\left(\mathscr{S}(G), \mathscr{S}^{\prime}(G)\right)$.

Theorem 10. $U$ is bijective.

Proof. If $v \in \mathscr{S}^{\prime}(C \times \hat{C})$ and $U(v)=0$, then $\left(v, F_{f g}\right)=0$ for all $f, g$ $\in \mathscr{S}(C)$, and so our above observations tell us that $v=0$.

For any $A \in \mathscr{L}\left(\mathscr{S}(C), \mathscr{S}^{\prime}(C)\right)$, consider the separately continuous bilinear functional $B_{A}:(f, g) \mapsto(A g, f)$. For any $\left(H_{1}, K_{1}\right)$ and $\left(H_{2}, K_{2}\right)$ in $\mathscr{A}, B_{A}$ is separately continuous when restricted to $\mathscr{S}\left(H_{1}, K_{1}\right) \otimes \mathscr{S}\left(H_{29}\right.$ $\left.K_{2}\right)$, and is thus jointly continuous, since $\mathscr{S}\left(H_{i}, K_{i}\right)$ is a Fréchet space for each $i$. Since both spaces $\mathscr{S}\left(H_{i}, K_{i}\right)$ are also nuclear, there exists some $v_{A}\left[H_{i}, K_{i}\right]$ in $\mathscr{S}^{\prime}\left(H_{1} \times H_{2}, K_{1} \times K_{2}\right)$ such that

$$
\left(v_{A}\left[H_{i}, K_{i}\right], f \otimes g\right)=(A g, f) \quad f \in \mathscr{S}\left(H_{1}, K_{1}\right), \quad g \in \mathscr{S}\left(H_{2}, K_{2}\right) .
$$

In the usual way we can now define a function $v_{A}: \mathscr{S}(C \times G) \rightarrow \mathbb{C}$ by setting $v_{A}(f)=\left(v_{A}\left[H_{i}, K_{i}\right], f\right)$ whenever $f \in \mathscr{S}\left(H_{1} \times H_{2}, K_{1} \times K_{2}\right)$. Then $v_{A} \in \mathscr{S}^{\prime}(C \times G)$ and $\left(v_{A}, f \otimes g\right)=(A g, f)$ for all $f, g \in \mathscr{S}(G)$. Thus we can define $u_{A} \in \mathscr{S}^{\prime}(C \times \hat{C})$ by setting $\left(u_{A}, \mathscr{F}_{2} R F\right)=\left(v_{A}, F\right)$ for all $F \in$ $\mathscr{S}(C \times G)$, and $u_{A}$ satisfies

$$
\left(U\left(u_{A}\right) g, f\right)=\left(u_{A}, F_{f g}\right)=\left(v_{A}, f \otimes g\right)=(A g, f)
$$

for all $f, g \in \mathscr{S}(C)$. Thus $U\left(u_{A}\right)=A$.

Composing $U$ with $\mathscr{F}_{\sigma}$ defined in the usual way, we obtain a bijection $W: \mathscr{S}^{\prime}(G) \rightarrow \mathscr{L}\left(\mathscr{S}(G), \mathscr{S}^{\prime}(C)\right)$. As in [5], we now let $\mathscr{E}$ denote the *-algebra of all continuous linear transformations from $\mathscr{S}(C)$ to itself that possess an adjoint, where $\mathscr{E}$ is regarded as a subspace of $\mathscr{L}\left(\mathscr{S}(G), \mathscr{S}^{\prime}(G)\right)$ in the usual way. Define $\mathcal{O}(G)$ to be the space of all distributions $u \in \mathscr{S}^{\prime}(G)$ with $W(u) \in \mathscr{E}$. Then $\mathcal{O}(G)$ possesses

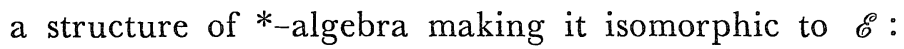

$$
W(u * v)=W(u) W(v) \quad W\left(u^{*}\right)=[W(u)]^{*}
$$

and simple calculations show that if $u \in \mathcal{O}(G)$, then $u^{*} \in \mathcal{O}(G)$ is given by the formula $\left(\overline{u^{*}, f}\right)=(u, \bar{f})$ for any $f \in \mathscr{S}(G)$. 
How does the algebra $\mathcal{O}(G)$ relate to the algebra $\mathcal{O}_{m}(G)$ found in $\S 4$, and how do the various convolutions $*$ and $*_{2}$ relate to each other? It is easy to see that $\mathscr{S}(G)$ belongs to both $\mathcal{O}(G)$ and $\mathcal{O}_{m}(G)$, so that in either case we have extended the old notion of the Weyl transformation. These questions may now be answered fully.

Theorem 11. $\mathcal{O}(G) \subseteq \mathcal{O}_{m}(G)$, and the convolutions $*$ and $*_{2}$ coincide on $\mathcal{O}(G)$. Indeed, it is easy to see that $\mathcal{O}(G)=\mathcal{O}_{m}(G) \cap \mathcal{O}_{m}(G) *$.

Proof. For any $v \in \mathcal{O}(G)$, we notice that $I \bar{\otimes} W(v) \in \mathscr{L}(\mathscr{S}(C \times C))$, and simple calculations show us that

$$
S F_{f, W(v) g}=\left[S \mathscr{F}_{2} R(I \bar{\otimes} W(v)) R^{-1} \mathscr{F}_{2}^{-1} S\right]\left(S F_{f_{g}}\right)=B(v)\left(S F_{f g}\right)
$$

for any $f, g \in \mathscr{S}(G)$, where $B(v) \in \mathscr{L}(\mathscr{S}(G))$. Now another simple calculation shows that $\left(\rho_{y} S F_{\overline{f g}}\right)(x)=F_{\overline{U(y) f}, g}(x)$, and so

$$
\left(\mathscr{F}_{{ }} v, \rho_{y} S F_{\overline{f g}}\right)=\langle U(y) f, W(v) g\rangle=\left[S F_{\bar{f}, W(v) g}\right](y)=\left[B(v)\left(S F_{\overline{f g}}\right)\right](y) .
$$

Since $\mathscr{S}(G \times G) \equiv \mathscr{S}(G) \bar{\otimes} \mathscr{S}(C)$, it follows that $\left\{S F_{f g} \mid f, g \in \mathscr{S}(C)\right\}$ spans a dense linear subspace of $\mathscr{S}(G \times \hat{C})$, so we deduce that $B(v) f=$ $\left(\mathscr{F}_{\sigma} v\right) \sigma_{1} f$ for all $f \in \mathscr{S}(C \times \hat{G})$. Hence $\mathscr{F}_{o} v \in \mathcal{O}_{c}(G)$, so $v \in \mathcal{O}_{m}(G)$, and $v^{*}{ }_{1} S \mathscr{F}_{\sigma} F_{f g}=S \mathscr{F}_{0} F_{f, W(v) g}$ for all $f, g \in \mathscr{S}(C)$.

Thus if $u, v \in \mathcal{O}(G)$, then $u, v \in \mathcal{O}_{m}(G)$ and

$$
\begin{aligned}
\left(u * v, S \mathscr{F}_{\sigma} F_{f g}\right) & =(W(u * v) g, f)=(W(u) W(v) g, f)=\left(u, S \mathscr{F}_{\sigma} F_{f, W(v) g}\right) \\
& =\left(u, v *_{1} S \mathscr{F}_{0} F_{f g}\right)=\left(u *_{2} v, S \mathscr{F}_{{ }_{0}} F_{f g}\right)
\end{aligned}
$$

for all $f, g \in \mathscr{S}(C)$, and hence $u * v=u *_{2} v$. The rest of the theorem follows trivially.

It should be noted that any linear map from $\mathscr{S}(G)$ to $\mathscr{S}^{\prime}(G)$ which is weakly continuous is automatically continuous, because $\mathscr{S}(G)$ is bornological, and hence a Mackey space [13]. If we had let $\widetilde{E}$ be the space of all maps in $\operatorname{End}(\mathscr{S}(C))$ which possessed an adjoint, then $\widetilde{\mathscr{E}}$ is a subspace of $\mathscr{L}\left(\mathscr{S}(C), \mathscr{S}^{\prime}(C)\right)$, and so any map $A \in \widetilde{\mathscr{E}}$ is a continuous linear map from $\mathscr{S}(C)$ to $\mathscr{S}(G)$, where the domain $\mathscr{S}(C)$ has its natural topology, and the range $\mathscr{S}(G)$ has the topology induced from $\mathscr{S}^{\prime}(C)$, namely the topology given by the seminorms $p_{f}(g)=|(g, f)|$ for all $f \in \mathscr{S}(C)$. Since these topologies are not equal, $\mathscr{E}$ is always a strict subspace of $\widetilde{E}$. 
The results in [5] show that there is essentially only one strictly irreducible representation of the ${ }^{*}$-algebra $\mathcal{O}(G)$, and this is provided by the Weyl transformation $W$. The algebra $\mathcal{O}(G)$ is in general very large-in the case $C=\boldsymbol{R}^{n}$ it contains all the natural physically interesting observables for the free particle-and so the methods of this paper provide a very powerful method of quantising physical systems whose phase space can be described in terms of a configuration space $G$.

\section{References}

[1] Bruhat, F. F., Distributions sur un groupe localement compact et applications à l'étude des représentations des groupes p-adiques, Bull. Soc. Math. France, 89 (1961), 43-75.

[2] Grothendieck, A., Produits tensoriels topologiques et espaces nucléaires, Mem. Amer. Math. Soc., 16 (1966).

[ 3 ] Hannabuss, K. C., Representations of nilpotent locally compact groups, J. Funct. Anal., 34.1 (1979), 146-165.

[4] Characters and contact transformations, Math. Proc. Cambridge Philos. Soc., 90 (1981), 465-476.

[5] Hennings, M. A., Fronsdal *-quantisation and the abstract inducing procedures of Fell and Rieffel, D. Phil. thesis, Oxford (1984).

[6] Hudson, R. L., Peck, S. N. \& Wilkinson, M. D., Fourier analysis on multiplier representations of locally compact abelian groups, Rep. Math. Phys., 19.1 (1984), 91-100.

[7] Igusa, J. -I., Harmonic analysis and theta functions, Acta Math., 120 (1968), 187-222.

[8] Kammerer, J. B., L'algèbre des opérateurs de multiplication du star-produit de $\mathbb{R}^{2 n}$, C. R. Acad. Sci. Paris Sér. I. Math., 298 (1984), 59-62.

[9] Kleppner, A., Multipliers on abelian groups, Math. Ann., 158 (1965), 11-34.

[10] Lunn, M., Some problems in group representation in quantum mechanics, D. Phil. thesis, Oxford (1969).

[11] Morris, S. A., Pontryagin duality and the structure of locally compact abelian groups, Lond. Math. Soc. LNS, 29 (1977).

[12] Reiter, H., Über den Satz von Weil-Cartier, Mh. Math., 86 (1978), 13-62.

[13] Schaefer, H. H., Topological vector spaces, Graduate Texts in Mathematics, SpringerVerlag (1971).

[14] Schwartz, L., Théorie des distributions, Hermann (1951). 
